\title{
Light-Like Boost of the Kerr Gravitational Field
}

\author{
C. Barrabès* \\ Laboratoire de Mathématiques et Physique Théorique, \\ CNRS/UMR 6083, Université F. Rabelais, 37200 TOURS, France \\ P. A. $\operatorname{Hogan}^{\dagger}$ \\ Mathematical Physics Department, \\ National University of Ireland Dublin, Belfield, Dublin 4, Ireland
}

\begin{abstract}
We describe light-like boosts of the Kerr gravitational field transverse and parallel to the symmetry axis. In the transverse case the boosted field is that of an impulsive gravitational wave having a line singularity displaced relative to its position if the rotation of the source were removed. The parallel boost is insensitive to the rotation of the source. The literature contains a number of diverse results for lightlike boosts of the Kerr gravitational field. Our conclusions confirm the correctness of the limits calculated by Balasin and Nachbagauer [Class. and Quantum Grav.13(1996),731]. To avoid any ambiguity our approach is centered on evaluating the light-like boost of the Riemann tensor for the Kerr space-time with the metric playing a secondary role.
\end{abstract}

To appear in Physical Review D

PACS number(s): $04.20 . \mathrm{Cv}$

\footnotetext{
*E-mail : barrabes@celfi.phys.univ-tours.fr

${ }^{\dagger}$ E-mail : phogan@ollamh.ucd.ie
} 


\section{Introduction}

Recently [1] a re-appraisal of the influential work by Aichelburg and Sexl [2] on the gravitational field of a static spherically symmetric body boosted to the speed of light was carried out. This involved putting the emphasis on the boosted gravitational field (Riemann tensor) with the metric playing a secondary role. Working with the Riemann tensor is a guaranteed way of ensuring that the boosted field is unambiguously determined because the Riemann tensor is gauge-invariant. This Riemann tensor centered approach was illustrated by calculating the boosted gravitational field of a static axially symmetric source. It is of some importance to apply this new point of view to the Kerr gravitational field, particularly in view of the many differing results which have been derived by authors who have considered what might be the analogue of the Aichelburg-Sexl result in this case [3] - 8]. Our results

are in general simpler and quite different to those obtained in the references cited with the notable exception that we find ourselves in agreement with the limit calculated by Balasin and Nachbagauer 4] (their equation (18) compared to our (2.29) below). These authors were the first to obtain this transverse light-like boosted Kerr gravitational field. They introduced a distributional energy-momentum tensor [3] for the Kerr space-time. This energy-momentum tensor was then subjected to a Lorentz boost and the light-like limit was taken. The metric of the space-time after the lightlike boost involves a single function which is obtained by solving a Poisson equation. The procedure is technically very impressive. However in the light of the existence of conflicting results in the other works cited, we feel that the limit calculated in [4] could benefit from being confirmed by the simpler derivation which we supply here.

\section{Boosted Kerr Field}

We begin with the Kerr line-element 9] in asymptotically rectangular Cartesian coordinates and time:

$$
d s^{2}=d s_{0}^{2}+\frac{2 m \bar{r}^{3}}{\bar{r}^{4}+a^{2} \bar{z}^{2}}\left[\frac{\bar{z}}{\bar{r}} d \bar{z}+\frac{(\bar{r} \bar{x}+a \bar{y})}{\bar{r}^{2}+a^{2}} d \bar{x}+\frac{(\bar{r} \bar{y}-a \bar{x})}{\bar{r}^{2}+a^{2}} d \bar{y}-d \bar{t}\right]^{2}
$$

with

$$
d s_{0}^{2}=d \bar{x}^{2}+d \bar{y}^{2}+d \bar{z}^{2}-d \vec{t}^{2} .
$$

We use units in which the speed of light $c=1$. Also the constants $m, a$ are the mass and the angular momentum per unit mass respectively of the 
source and $\bar{r}$ is a function of $\bar{x}, \bar{y}, \bar{z}$ given by

$$
\frac{\bar{x}^{2}+\bar{y}^{2}}{\bar{r}^{2}+a^{2}}+\frac{\bar{z}^{2}}{\bar{r}^{2}}=1 \text {. }
$$

Thus in coordinates $\bar{x}^{\mu}=(\bar{x}, \bar{y}, \bar{z}, \bar{t})$ the metric tensor components have the Kerr-Schild form [10] 11]

$$
\bar{g}_{\mu \nu}=\bar{\eta}_{\mu \nu}+2 \bar{H} \bar{k}_{\mu} \bar{k}_{\nu}
$$

with

$$
\begin{aligned}
\eta_{\mu \nu} d \bar{x}^{\mu} d \bar{x}^{\nu} & =d \bar{x}^{2}+d \bar{y}^{2}+d \bar{z}^{2}-d \bar{t}^{2} \\
\bar{k}_{\mu} d \bar{x}^{\mu} & =\frac{\bar{z}}{\bar{r}} d \bar{z}+\frac{(\bar{r} \bar{x}+a \bar{y})}{\bar{r}^{2}+a^{2}} d \bar{x}+\frac{(\bar{r} \bar{y}-a \bar{x})}{\bar{r}^{2}+a^{2}} d \bar{y}-d \bar{t} \\
\bar{H} & =\frac{m \bar{r}^{3}}{\bar{r}^{4}+a^{2} \bar{z}^{2}},
\end{aligned}
$$

with the covariant vector field $\bar{k}_{\mu}$ null with respect to $\bar{g}_{\mu \nu}$ and thus null with respect to the auxiliary Minkowskian metric tensor $\bar{\eta}_{\mu \nu}$. Later we shall be transforming to unbarred coordinates $\{x, y, z, t\}$.

We shall require the coordinate components $\bar{R}_{\mu \nu \rho \sigma}$ of the Riemann curvature tensor calculated from the metric tensor (2.4). These are conveniently given in terms of the complex tensor

$$
{ }^{+} \bar{R}_{\mu \nu \rho \sigma}=\bar{R}_{\mu \nu \rho \sigma}+i^{*} \bar{R}_{\mu \nu \rho \sigma}
$$

where the left dual of the Riemann tensor (since the Kerr space-time is a vacuum space-time the left and right duals of the Riemann tensor are equal) is defined by ${ }^{*} \bar{R}_{\mu \nu \rho \sigma}=\frac{1}{2} \bar{\eta}_{\mu \nu \alpha \beta} \bar{R}_{\rho \sigma}^{\alpha \beta}$ with $\bar{\eta}_{\mu \nu \alpha \beta}=\sqrt{-\bar{g}} \epsilon_{\mu \nu \alpha \beta}, \bar{g}=\operatorname{det}\left(\bar{g}_{\mu \nu}\right)$ and $\epsilon_{\mu \nu \alpha \beta}$ is the Levi-Civita permutation symbol. We find after a lengthy calculation, using many of the calculations in [1], that for the Kerr spacetime

$$
{ }^{+} \bar{R}_{\mu \nu \rho \sigma}=-\frac{m \bar{r}^{3}}{\left(\bar{r}^{2}+i a \bar{z}\right)^{3}}\left\{\bar{g}_{\mu \nu \rho \sigma}+i \epsilon_{\mu \nu \rho \sigma}+3 \bar{W}_{\mu \nu} \bar{W}_{\rho \sigma}\right\},
$$

with

$$
\bar{g}_{\mu \nu \rho \sigma}=\bar{g}_{\mu \rho} \bar{g}_{\nu \sigma}-\bar{g}_{\mu \sigma} \bar{g}_{\nu \rho},
$$

and the bivector $\bar{W}_{\mu \nu}=-\bar{W}_{\nu \mu}$ is given by the $2-$ form

$$
\begin{aligned}
\frac{1}{2} \bar{W}_{\mu \nu} d \bar{x}^{\mu} \wedge d \bar{x}^{\nu} & =\frac{\bar{r}}{\left(\bar{r}^{2}+i a \bar{z}\right)}[\bar{x}(d \bar{x} \wedge d \bar{t}-i d \bar{y} \wedge d \bar{z})+\bar{y}(d \bar{y} \wedge d \bar{t} \\
& -i d \bar{z} \wedge d \bar{x})+(\bar{z}+i a)(d \bar{z} \wedge d \bar{t}-i d \bar{x} \wedge d \bar{y})] .
\end{aligned}
$$


We now consider a Lorentz boost in the $-\bar{x}$ direction with 3 -velocity $v<1$. Thus we transform from coordinates $\bar{x}^{\mu}=(\bar{x}, \bar{y}, \bar{z}, \bar{t})$ to coordinates $x^{\mu}=(x, y, z, t)$ given by

$$
\bar{x}=\gamma(x-v t), \quad \bar{y}=y, \quad \bar{z}=z, \quad \bar{t}=\gamma(t-v x),
$$

with $\gamma=\left(1-v^{2}\right)^{-1 / 2}$. The components ${ }^{+} \bar{R}_{\mu \nu \rho \sigma}$ are transformed to ${ }^{+} R_{\mu \nu \rho \sigma}$. The transformations naturally divide into three sets of equations. The first set has $\gamma^{2}$ as a factor on each right hand side and is

$$
\begin{aligned}
{ }^{+} R_{1 A 1 B} & =\gamma^{2}\left({ }^{+} \bar{R}_{1 A 1 B}+v^{+} \bar{R}_{1 A B 4}+v^{+} \bar{R}_{1 B A 4}+v^{2+} \bar{R}_{A 4 B 4}\right), \\
{ }^{+} R_{1 A 4 B} & =\gamma^{2}\left({ }^{+} \bar{R}_{1 A 4 B}+v^{+} \bar{R}_{1 A B 1}+v^{+} \bar{R}_{4 A B 4}+v^{2+} R_{4 A 1 B}\right), \\
{ }^{+} R_{4 A 4 B} & =\gamma^{2}\left(+\bar{R}_{4 A 4 B}+v^{+} \bar{R}_{4 A B 1}+v^{+} \bar{R}_{4 B A 1}+v^{2+} \bar{R}_{A 1 B 1}\right)
\end{aligned}
$$

where the subscripts $A, B$ take values 2,3 . The second set has $\gamma$ as a factor on each right side and reads

$$
\begin{aligned}
{ }^{+} R_{1 A 14} & =\gamma\left({ }^{+} \bar{R}_{1 A 14}+v^{+} \bar{R}_{14 A 4}\right), \\
{ }^{+} R_{14 A 4} & =\gamma\left({ }^{+} \bar{R}_{14 A 4}+v^{+} \bar{R}_{1 A 14}\right), \\
{ }^{+} R_{1 A B C} & =\gamma\left({ }^{+} \bar{R}_{1 A B C}+v^{+} \bar{R}_{A 4 B C}\right), \\
{ }^{+} R_{4 A B C} & =\gamma\left({ }^{+} \bar{R}_{4 A B C}+v^{+} \bar{R}_{A 1 B C}\right) .
\end{aligned}
$$

Finally we have the set of transformed components which do not involve the $\gamma$ factor:

$$
{ }^{+} R_{1414}={ }^{+} \bar{R}_{1414}, \quad{ }^{+} R_{1423}={ }^{+} \bar{R}_{1423}, \quad{ }^{+} R_{2323}={ }^{+} \bar{R}_{2323} .
$$

In the right hand sides of (2.13)-(2.15) the components ${ }^{+} \bar{R}_{\mu \nu \rho \sigma}$ are substituted from (2.9) and then the resulting quantities are written in terms of the coordinates $\{x, y, z, t\}$ using (2.12). There is one relation between these components:

$$
{ }^{+} R_{1234}+{ }^{+} R_{1423}+{ }^{+} R_{1342}=0 \text {. }
$$

We now in effect "boost" the Kerr source to the speed of light transverse to its symmetry axis (the $z$-axis). This is analogous to the Aichelburg-Sexl 2] boost of the Schwarzschild field (to which it specialises when $a=0$ ) and is achieved by taking the limit $v \rightarrow 1$ above. The gravitational field of the boosted source is given by

$$
{ }^{+} \tilde{R}_{\mu \nu \rho \sigma}=\lim _{v \rightarrow 1}{ }^{+} R_{\mu \nu \rho \sigma} .
$$


Since the Kerr space-time is a vacuum space-time its Ricci tensor $\bar{R}_{\mu \nu}=0$ and hence the Lorentz transformed Ricci tensor $R_{\mu \nu}=0$ and therefore $\tilde{R}_{\mu \nu}=$ 0 . In this limit the rest-mass $m \rightarrow 0$ as $\gamma \rightarrow \infty$ in such a way that the relative energy $m \gamma=p$ (say) remains finite. For a source with multipole moments, all moments behave like the monopole moment $m$ in the limit $v \rightarrow 1$ in the case of the transverse boost (for further explanation of this point see the calculations described in section IV of [1]). Thus the parameter $a$ is unaffected by the boost in this case. For a boost parallel to the symmetry axis the situation, which is quite different, is discussed in the next section. To evaluate the limit (2.17) explicitly we need the following:

$$
\lim _{v \rightarrow 1} \frac{\gamma \bar{r}^{3}}{\left(\bar{r}^{2}+i a \bar{z}\right)^{3}}=\frac{2 \delta(x-t)}{y^{2}+(z+i a)^{2}},
$$

and

$$
\lim _{v \rightarrow 1} \frac{\gamma \bar{r}^{5}}{\left(\bar{r}^{2}+i a \bar{z}\right)^{5}}=\frac{4}{3} \frac{\delta(x-t)}{\left(y^{2}+(z+i a)^{2}\right)^{2}},
$$

where $\delta(x-t)$ is the Dirac delta function singular on $x=t$. To establish (2.18) we note that (2.3) and (2.12) can be used to write

$$
\frac{\gamma \bar{r}^{3}}{\left(\bar{r}^{2}+i a \bar{z}\right)^{3}}=\frac{1}{\left(y^{2}+(z+i a)^{2}\right)} \frac{\partial}{\partial x}\left(\frac{(x-v t) \bar{R}}{\bar{R}^{2}+i \gamma^{-2} a z}\right) .
$$

The quantity $\bar{R}$ is defined as follows: first we have from (2.3) that

$$
\bar{r}^{2}=\frac{1}{2}\left\{r^{2}-a^{2}+\sqrt{\left(r^{2}-a^{2}\right)^{2}+4 a^{2} z^{2}}\right\},
$$

with $r^{2}=\bar{x}^{2}+\bar{y}^{2}+\bar{z}^{2}=\gamma^{2}(x-v t)^{2}+y^{2}+z^{2}$. Thus we have

$$
r=\gamma R, \quad R=\sqrt{(x-v t)^{2}+\gamma^{-2}\left(y^{2}+z^{2}\right)},
$$

and so (2.21) yields

$$
\bar{r}=\gamma \bar{R}, \quad \bar{R}^{2}=\frac{1}{2}\left\{R^{2}-\gamma^{-2} a^{2}+\sqrt{\left(R^{2}-\gamma^{-2} a^{2}\right)^{2}+4 \gamma^{-4} a^{2} z^{2}}\right\},
$$

giving us $\bar{R}$. With $\bar{R}$ and $R$ now defined we see from (2.20) that

$$
\lim _{v \rightarrow 1} \frac{\gamma \bar{r}^{3}}{\left(\bar{r}^{2}+i a \bar{z}\right)^{3}}=\frac{1}{\left(y^{2}+(z+i a)^{2}\right)} \frac{\partial}{\partial x}\left(\frac{x-t}{|x-t|}\right) .
$$

If $\theta(x-t)$ is the Heaviside step function which is equal to unity if $x>t$ and is zero if $x<t$ then

$$
\frac{x-t}{|x-t|}=2 \theta(x-t)-1
$$


and since $\partial \theta / \partial x=\delta(x-t)$ we recover (2.18) from (2.24).

By (2.3) and (2.12) we have

$$
\frac{\partial \bar{r}}{\partial y}=\frac{y \bar{r}^{3}}{\bar{r}^{4}+a^{2} \bar{z}^{2}}
$$

and using this we find that (2.19) follows from (2.18) by differentiating (2.18) with respect to $y$ and taking $y \neq 0$. When $a=0$ we note that (2.18) and (2.19) reduce to $(2.10)$ and (3.5) respectively of [1]

We now evaluate the limits (2.17). As an illustration we find

${ }^{+} \tilde{R}_{1212}=\lim _{v \rightarrow 1}\left[\frac{3 m \gamma^{2} \bar{r}^{5}}{\left(\bar{r}^{2}+i a \bar{z}\right)^{5}}\{z+i(a+y)\}^{2}\right]=4 p\left[\frac{z+i(a+y)}{y^{2}+(z+i a)^{2}}\right]^{2} \delta(x-t)$,

by (2.19). After some algebra this can be rewritten as

$$
+\tilde{R}_{1212}=4 p\left[\frac{z+i(y-a)}{z^{2}+(y-a)^{2}}\right]^{2} \delta(x-t),
$$

and if

$$
H=2 p \log \left\{(y-a)^{2}+z^{2}\right\} \delta(x-t)
$$

we finally have

$$
{ }^{+} \tilde{R}_{1212}=\left(H_{y y}-i H_{y z}\right) .
$$

The subscripts on $H$ denote second partial derivatives. In similar fashion we find that $\tilde{R}_{\mu \nu \rho \sigma} \equiv 0$ except for

$$
\begin{aligned}
{ }^{+} \tilde{R}_{1212} & ={ }^{+} \tilde{R}_{2424}=-{ }^{+} \tilde{R}_{1313}=-{ }^{+} \tilde{R}_{3434}={ }^{+} \tilde{R}_{3134}=-{ }^{+} \tilde{R}_{2124} \\
& =\left(H_{y y}-i H_{y z}\right)
\end{aligned}
$$

and

$$
\begin{aligned}
{ }^{+} \tilde{R}_{1213} & ={ }^{+} \tilde{R}_{2434}=-{ }^{+} \tilde{R}_{3124}=-{ }^{+} \tilde{R}_{2134} \\
& =i\left(H_{y y}-i H_{y z}\right)
\end{aligned}
$$

When the Lorentz transformation (2.12) is applied to the line-element (2.1) and the limit $v \rightarrow 1$ taken we find that if $x-t>0$ then

$$
\lim _{v \rightarrow 1} d s^{2}=d x^{2}+d y^{2}+d z^{2}-d t^{2}+\frac{8 p}{x-t}(d x-d t)^{2},
$$

and if $x-t<0$ then

$$
\lim _{v \rightarrow 1} d s^{2}=d x^{2}+d y^{2}+d z^{2}-d t^{2} .
$$


For $x-t>0$ we can write (2.33) in the form

$$
d s_{+}^{2}=d y_{+}^{2}+d z_{+}^{2}+2 d u d v_{+}
$$

with

$$
\begin{aligned}
& y_{+}=y \quad z_{+}=z, \quad u=x-t, \\
& v_{+}=\frac{1}{2}(x+t)+4 p \log (x-t) .
\end{aligned}
$$

For $x-t<0$ we can write (2.34) in the form

$$
d s_{-}^{2}=d y_{-}^{2}+d z_{-}^{2}+2 d u d v_{-}
$$

with

$$
\begin{aligned}
& y_{-}=y \quad z_{-}=z, \quad u=x-t \\
& v_{-}=\frac{1}{2}(x+t) .
\end{aligned}
$$

From (2.35) and (2.37) we see that $u=0$ is a null hyperplane in Minkowskian space-time. The line-elements (2.35) and (2.37) are consistent with having a delta function in the Riemann curvature tensor which is singular on $x=t$ provided the two halves of Minkowskian space-time , $x>t$ and $x<t$, are attached on $x=t$ with

$$
y_{+}=y_{-}, \quad z_{+}=z_{-}, \quad v_{+}=F\left(v_{-}, y_{-}, z_{-}\right)
$$

for some function $F$ defined on $x=t$ for which $\partial F / \partial v_{-} \neq 0$ [13]. The particular function $F$ which gives rise to the coefficients of the delta function in the Riemann tensor components listed in (2.31) and (2.32) can be calculated from the formulas given in [1] (and originally derived in 13). It is easily found to be

$$
v_{+}=F=v_{-}+2 p \log \left\{(y-a)^{2}+z^{2}\right\} .
$$

The signal with history $x=t$ is an impulsive gravitational wave with a delta function profile and which is, in addition, singular on the null geodesic generator $y=a, z=0$ of the null hyperplane $x=t$. This is identical to the light-like boosted Schwarzschild field [2] except that the singular generator is shifted from $y=0, z=0$ in that case to $y=a, z=0$. The physical significance of this shifted generator (the imprint in the boosted field of the rotation of the source of the original Kerr gravitational field) can be elucidated, for example, by studying the deflection of highly relativistic particles in the Kerr gravitational field [12]. 
Once $F$ in (2.40) is known the line-element of the space-time can be constructed in coordinates in which the metric tensor is continuous across $x=t[13$. For the light-like boosted Kerr field the resulting line-element can then be transformed into Kerr-Schild form at the expense of introducing the delta function into the metric tensor. This line-element is

$$
d s^{2}=d x^{2}+d y^{2}+d z^{2}-d t^{2}-2 H(d x-d t)^{2},
$$

with $H$ given by (2.29). The corresponding Riemann tensor (2.31) and (2.32) is type $\mathrm{N}$ in the Petrov classification with degenerate principal null direction given via the 1 -form $d x-d t$.

\section{Discussion}

The light-like boost of the Kerr gravitational field described above involves a boost transverse to the symmetry axis of the Kerr source. To complete the picture we consider briefly here the simpler case of a light-like boost in the $-\bar{z}$-direction which is parallel to the symmetry axis. To motivate our approach to this we expand $F$ given in (2.40) for the transverse light-like boost in powers of the parameter $a$. Thus we arrive at

$$
F=v_{-}+2 \sum_{l=0}^{\infty}(-1)^{l} \frac{p_{l}}{l !} \frac{\partial^{l}}{\partial y^{l}}\left(\log \left(y^{2}+z^{2}\right)\right)
$$

with $p_{l}=p a^{l}$ for $l=0,1,2, \ldots$. This is similar to the matching function $F$ encountered in the transverse light-like boost of a static axially symmetric multipole field [1] whose original multipole moments, before taking the limit $v \rightarrow 1$, were

$$
A_{l}=m a^{l}, \quad l=0,1,2, \ldots,
$$

and with $m=p \gamma^{-1}$. For the light-like boost in the $-\bar{z}$-direction (the direction of the symmetry axis) the analogy with the multipole field [1] suggests that we take $a=\gamma^{-1} \hat{a}$ as $v \rightarrow 1$ in this case, aswell as taking $m=p \gamma^{-1}$. Then (3.2) is replaced by

$$
A_{l}=\gamma^{-l-1} p_{l}, \quad p_{l}=p \hat{a}^{l},
$$

for $l=0,1,2, \ldots$ as in the case of a light-like boost parallel to the symmetry axis in [1]. Now make the Lorentz transformation

$$
\bar{x}=x, \quad \bar{y}=y, \quad \bar{z}=\gamma(z-v t), \quad \bar{t}=\gamma(t-v z) .
$$


The components ${ }^{+} \bar{R}_{\mu \nu \rho \sigma}$ in (2.9) are transformed to ${ }^{+} R_{\mu \nu \rho \sigma}$. In this case the equations (2.13) and (2.14) are replaced by the same equations, with the subscript 1 replaced by the subscript 3 and with $A, B, C$ each taking the values 1,2. Also the subscripts 1 and 3 are interchanged in equations (2.15). The relation (2.16) continues to hold. Now (2.19) is replaced by

$$
\lim _{v \rightarrow 1} \frac{\gamma \bar{r}^{5}}{\left(\bar{r}^{2}+i a \bar{z}\right)^{5}}=\lim _{v \rightarrow 1} \frac{\gamma \bar{r}^{5}}{\left\{\bar{r}^{2}+i \hat{a}(z-v t)\right\}^{5}}=\lim _{v \rightarrow 1} \frac{\gamma^{-4}}{\tilde{R}^{5}}=\frac{4}{3} \frac{\delta(z-t)}{\left(x^{2}+y^{2}\right)^{2}}
$$

where $\tilde{R}=\sqrt{(z-v t)^{2}+\gamma^{-2}\left(x^{2}+y^{2}\right)}$. The last equality in (3.5) is derived as Eq.(3.5) in [1]. Calculating $+\tilde{R}_{\mu \nu \rho \sigma}$ in (2.17) in this case we find that the non-identically vanishing components are

$$
\begin{aligned}
{ }^{+} \tilde{R}_{2323} & ={ }^{+} \tilde{R}_{2424}=-{ }^{+} \tilde{R}_{2324}=-{ }^{+} \tilde{R}_{3131}=-{ }^{+} \tilde{R}_{1414}={ }^{+} \tilde{R}_{1314} \\
& =\frac{4 p(x-i y)^{2}}{\left(x^{2}+y^{2}\right)^{2}} \delta(z-t)
\end{aligned}
$$

and

$$
{ }^{+} \tilde{R}_{3231}={ }^{+} \tilde{R}_{2414}=-{ }^{+} \tilde{R}_{1324}=-{ }^{+} \tilde{R}_{2314}=-\frac{4 i p(x-i y)^{2}}{\left(x^{2}+y^{2}\right)^{2}} \delta(z-t) .
$$

This is the same field as the light-like boosted Schwarzschild field derived by Aichelburg and Sexl 2. The generalisation of the calculations given here to a light-like boost of the Kerr gravitational field in an arbitrary direction involves tedious algebra but is straightforward and would result in an explicit limit for the Riemann tensor.

\section{References}

[1] C. Barrabès and P. A. Hogan, Phys. Rev. D64, 044022 (2001).

[2] P. C. Aichelburg and R. U. Sexl, Gen. Relativ. Gravit. 12, 303 (1971).

[3] H. Balasin and H. Nachbagauer, Class. Quantum Grav. 12, 707 (1995).

[4] H. Balasin and H. Nachbagauer, Class. Quantum Grav. 13, 731 (1996).

[5] A. Burinskii and G. Magli, Phys. Rev. D61, 044017 (2000).

[6] V. Ferrari and P. Pendenza, Gen. Relativ. Gravit. 22, 1105 (1990).

[7] C. O. Lousto and N. Sanchez, Nucl. Phys. B355, 231 (1991). 
[8] C. O. Lousto and N. Sanchez, Nucl. Phys. B383, 377 (1992).

[9] R. P. Kerr, Phys. Rev. Letters 11, 237 (1963).

[10] R. P. Kerr and A. Schild, Atti del Convegno sulla Relatività Generale: Problemi dell Energia e Onde Gravitazionali (Anniversary Volume, Fourth Centenary of Galileo's Birth), G. Barbéra, Ed. (Firenze, 1965), p. 173; Applications of Nonlinear Partial Differential Equations in Mathematical Physics, Proceedings of Symposia in Applied Mathematics (American Mathematical Society, Providence, R.I., 1965), vol. XVII, p. 199.

[11] G. C. Debney, R. P. Kerr and A. Schild, J. Math. Phys. 10, 1842 (1969).

[12] C. Barrabès and P. A. Hogan, "The Deflection of Highly Relativistic Particles in a Gravitational Field" (preprint) 2002.

[13] C. Barrabès and P. A. Hogan, Int. J. Mod. Phys. D10, 711 (2001). 